\title{
¿Podría estar contribuyendo el cooperativismo a fijar la población en el territorio de Andalucía?
}

\section{Lidia Valiente Palma}

RESUMEN: En Andalucía las zonas rurales presentan marcadas desventajas frente a las urbanas, como son la falta de infraestructura, la escasez de servicios básicos o la falta de dinámicas económicas que provocan éxodo rural, entre otras, lo que genera desequilibrios territoriales en la región. Este éxodo rural, a su vez, influye como limitador de la generación de estas dinámicas, provocando un creciente envejecimiento de la población y mermando las oportunidades de desarrollo del territorio. Ante esta problemática, las cooperativas pueden tener un papel significativo por su capacidad para fijar la población en los territorios. A partir de lo anterior, el objetivo del presente trabajo consiste en determinar si las sociedades cooperativas en Andalucía podrían estar contribuyendo a mantener la población en el territorio, sobre todo rurales, en mayor grado que las empresas mercantiles. Para ello, se realizan análisis exploratorios y de regresiones espaciales al objeto de identificar si un mayor peso de cooperativas o de empresas mercantiles, a nivel municipal, se traduce en una menor tasa de emigraciones. Entre los resultados destaca que existen indicios acerca de que el cooperativismo podría estar suponiendo una oportunidad para evitar las emigraciones de mujeres y jóvenes en los territorios rurales.

PALABRAS CLAVE: Andalucía, sociedades cooperativas, territorio, emigraciones, regresión espacial.

CLAVES ECONLIT: C21, 018, P13, R23.

Cómo citar este articulo / How to cite this article: VALIENTE, L. (2019): “¿Podría estar contribuyendo el cooperativismo a fijar la población en el territorio de Andalucía?", CIRIEC-España, Revista de Economía Pública, Social y Cooperativa, 97, 49-74. DOI: 10.7203/CIRIEC-E.97.13046.

Correspondencia: Lidia Valiente Palma, Universidad de Cádiz, lidia.valiente@uca.es. 


\section{EXPANDED ABSTRACT}

\section{Is cooperativism helping to keep the population in Andalusia?}

\section{Rationale and current interest in topic}

Rural areas in Andalusia present clear disadvantages as compared to urban areas, such as lack of infrastructure, scarcity of basic services or lack of economic dynamics, causing a rural exodus and thus giving rise to territorial imbalances in the region. This rural exodus, in turn, has a limiting influence on the generation of economic dynamics, resulting in an increasingly ageing population and reducing opportunities for territorial development.

In the face of this problem, cooperatives -societies of the social economy- may play a significant role in terms of their capacity for attaching a population to its territory, due to the existence of a relationship with the surroundings in which they operate, as demonstrated by the available literature on this topic (García-Gutiérrez, 1999; Buendía and García, 2003; Coque, 2005; Mozas and Bernal, 2006; Cuñat and Coll, 2007; Puentes and Velasco, 2009; Calvo and González, 2011; Demoustier, 2011; Draperi, 2014; Guzmán, Santos and Barroso, 2016; Pérez and Valiente, 2017).

Moreover, there is significant cooperativism in this region (with $19 \%$ of cooperatives and $18 \%$ of the employment these generate nationwide located in Andalusia) as well as consolidated institutional recognition of these organisations (as evinced by the laws which regulate them, as well as by the Pactos Andaluces por la Economía Social [Andalusia Agreements for the Social Economy] or by the various support programs for cooperative organisations). This represents an opportunity for the creation of economic dynamics and for attaching the population to its territory through the promotion of social economy societies.

\section{Objectives}

The main objective of this work consists in determining whether cooperative societies in Andalusia may be contributing to the maintenance of the region's population, especially in rural areas, to a greater extent than mercantile businesses.

In addition to confirming whether this occurs throughout the entire population, we have separated out the populations of women and young people, due to the difficulties currently faced by these groups in joining the labour market (the main reason for emigration, especially in rural areas) and to their strategic interest in terms of consolidating the region's population, passing the inter-generational torch in local economic activities. 
A comparison of cooperative societies with mercantile businesses is carried out in order to lay the foundations for the establishment of strategies and policies for the specific promotion of cooperativism, in the event that the behaviour seen in these social economy organisations differs from that of mercantile businesses.

\section{Methodology}

In working towards the stated objective, and after an exploratory analysis of the variables used, spatial econometrics techniques were applied which take into account the location of the data in a given geographic space for the variables under study: spatial distribution and autocorrelation analysis graphsand the application of spatial regression. Calculation of the models was carried out with GeoDa software.

With respect to the variables used, these were the following: percentage of total emigration, percentage of emigration of young people and percentage of emigration of women were considered as dependent, proxy variables for the attachment of population to territory; the number of cooperatives out of the total number of businesses was established, as well as the number of mercantile businesses (public limited companies and limited liability companies) out of the total number of businesses, expressed as percentages, served as explanatory variables. These variables were obtained for the various Andalusian municipalities (a total of 770) for 2015. In addition, for the purpose of determining whether differences exist in emigration between rural and non-rural municipalities, a dummy variable was added, termed rural municipality or territory.

\section{Results, practical conclusions and research limitations}

The results obtained provide indications that cooperativism may be contributing to an attachment of the population to its territory (as compared to mercantile businesses, where a significant relationship was not seen), since the regression analyses demonstrated that the variable for percentage of cooperative societies is significant in explaining the variability seen in emigration. Moreover, both show an inverse relationship. The same was obtained when the said dependent variables used were percentage of emigration of women and young people. In other words, the proportion of cooperatives inversely influence these emigration rates, which is not the case for mercantile businesses.

Based on the aforementioned and given that, on the one hand, difficulties in attaching a population to its territory are further complicated in rural Andalusian municipalities, especially for women and young people and, on the other hand, cooperativism inversely influences emigration from rural municipalities, the creation of quality, sustainable employment through cooperatives societies of social economymay represent an opportunity for reducing problems of depopulation in these areas. 
Another result obtained is that the cooperative business culture may be spread to neighbouring regions to a lesser extent than that of the conventional business sector. If cooperativism can contribute to an attachment of population to territory, the application of specific policies and strategies for reducing emigration from rural areas -promoting the development of cooperative societies for the creation of quality, sustainable employment and thus spreading cooperative culture to a greater extentbecomes at once a challenge and an opportunity for Andalusia. From another perspective, business synergies between cooperativism and conventional businesses may be encouraged, making the most of the opportunity offered by the latter for spreading their culture to neighbouring regions to a greater degree than cooperativism. In this way, spreading the principles and values of cooperativism in the Andalusian region can be achieved at the same time as contributing to an attachment of population to territory, through a potential business inter-cooperation.

Among the conclusions drawn, one also finds the need to carry out specific strategies and policies for promoting cooperativism in Andalusia, derived from results showing more heterogeneous behaviour in the region than seen in the conventional business sector.

With regard to the limitations of this work, it should be noted that, in the first place, the analysis carried out is static in nature, based on specific moment in time (the year 2015), which represents a limitation for analysing the evolutionary dynamic followed by both cooperatives societies and emigration in Andalusia. In the second place, percentage of emigration was taken as a proxy or indicative variable, with respect to the difficulty in attaching a population to its territory. However, the presence of other factors must be taken into account: it may be that the percentage of emigration is low or null as a result of considerable ageing in the population, such that depopulation or difficulty in attaching population to territory would be caused, rather, by a lack of demographic growth.

This work has established a number of future lines of research stemming in part from the very limitations indicated above, among which are the analysis of the evolutionary dynamic between cooperativism and emigration for the purpose of identifying the relationship these have followed over time; as well as consideration of other factors indicating capacity for attaching population to territory, such as the previously mentioned rate of demographic growth.

KEYWORDS: Andalusia, cooperative societies, territory, emigration, spatial regression. 


\section{Introducción}

La globalización, desde una perspectiva económica, está generando territorios ganadores y perdedores (Colletis y Pecqueur, 1993) y acentuando la segregación entre zonas rurales y urbanas debido al progresivo atraso relativo que han sufrido los primeros (Silva, 2002). Siguiendo a Silva (2002: 104), la mundialización de la economía y la creciente generalización de las nuevas tecnologías de la información, han hecho vulnerables a estos territorios rurales ya que, a pesar de ser más accesibles, están expuestos a una competencia exterior significativa. Esta vulnerabilidad se manifiesta, entre otros aspectos, mediante la dificultad para fijar la población al territorio.

Centrando la atención en la comunidad autónoma de Andalucía, región con un marcado atraso socioeconómico respecto a otros territorios españoles, hay que destacar que también presenta disparidades dentro del propio territorio; es decir, las desigualdades en Andalucía desde diferentes dimensiones -sociales, económicas, medioambientales, etc.- tienen lugar tanto respecto al resto de comunidades autónomas como a nivel intrarregional, donde existen importantes brechas entre zonas urbanas y rurales. Son diversos los trabajos (Torres y Ojeda, 2004; Caravaca, González y Mendoza, 2007; Méndez, Melero y Calatrava, 2008; Zoido et al. 2009; Pita y Pedregal, coords. 2011; Sánchez, Gallardo y Ceña, 2014; entre otros) que han puesto de manifiesto esta diversidad territorial presente en la región desde diferentes perspectivas y objetos de estudio.

En línea con lo anterior, desde una perspectiva cuantitativa, según la Ley de Desarrollo Sostenible del Medio Rural (LDSMR), que considera que un municipio es rural de pequeño tamaño cuando éste posee una población inferior a 5.000 habitantes, hay que destacar que más del $66 \%$ del territorio de Andalucía puede ser considerado como rural (Gráfico1). Estas zonas se ven afectadas por un despoblamiento significativo, consecuencia de la inexistencia de oportunidades laborales para sus habitantes. Este éxodo rural, a su vez, influye como limitador de la generación de dinámicas económicas, provocando un creciente envejecimiento de la población y mermando las oportunidades de desarrollo del territorio (Juliá y Marí, 2002; Soler, 2009; Cabanes y Gómez, 2014), pues estos grupos poblacionales constituyen un factor clave en el proceso productivo y son los principales receptores de los bienes y servicios producidos. 


\section{Gráfico1. Mapa de municipios rurales andaluces según la Ley de Desarrollo Sostenible del Medio Rural (1: municipio rural, 0 : municipio no rural)}

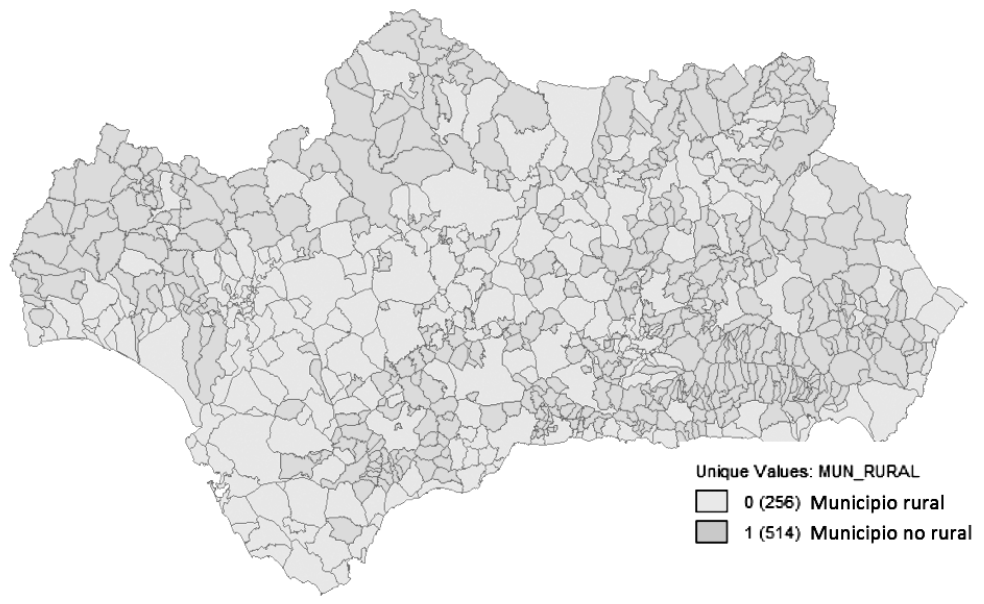

FUENTE: Elaboración propia.

Ante la problemática descrita, determinados colectivos como mujeres y jóvenes que actualmente presentan ciertas dificultades de acceso al mercado laboral, principal motivo de emigración, tienen un papel relevante, por lo que actuar sobre estos grupos son clave para la fijación de la población al territorio, sobre todo rurales, y salvar las dificultades que ocasiona un alto grado de envejecimiento. De ahí la importancia de la creación de oportunidades laborales para estos colectivos (García, 2004) a partir de las potencialidades de desarrollo de los territorios.

Por otro lado, relacionando las sociedades cooperativas con el territorio y la población, estas organizaciones de economía social están capacitadas para reforzar el vínculo de las economías y sociedades con "lo local": se caracterizan por una serie de principios y valores de actuación, entre los que se encuentra el compromiso con la comunidad local, manifestándose éste por medio de las actividades que realiza como las de reinserción social o promoción del emprendimiento social y colectivo (Novkovic, 2008), la respuesta a nuevas necesidades sociales mediante los Nuevos Yacimientos de Empleo (NYE) (Vilallonga, 2002; Tortosa, De Pablo y Uribe, 2018) o la creación de empleo sostenible (Pérez y Valiente, 2018), contribuyendo a disminuir las desigualdades sociales de los territorios (Burgués, Martin y Santa Cruz, 2013).

En definitiva y como consecuencia de estas ventajas o potencialidades, las sociedades cooperativas pueden contribuir a fijar la población al territorio (Coque, 2005; Mozas y Bernal, 2006; Buendía y Côté, 2014; Esteban, Pérez y Gargallo, 2018), lo que se analiza en el presente trabajo para el caso de Andalucía. 
Además de lo señalado arriba, el centrar el interés de este estudio en la comunidad autónoma de Andalucía se debe a la presencia de un cooperativismo significativo en esta región, ya que el 19\% de las cooperativas y el $18 \%$ del empleo que éstas generan a nivel nacional se encuentran en Andalucía. A ello hay que añadir que existe un reconocimiento institucional consolidado de estas organizaciones que se evidencia por las leyes reguladoras de éstas, los Pactos Andaluces por la Economía Social o los diferentes programas de apoyo a sus organizaciones.

Partiendo de la situación anterior de Andalucía y de la literatura sobre las ventajas de las sociedades cooperativas para fijar la población al territorio, el objetivo del presente trabajo consiste en determinar si dichas sociedades en esta región podrían estar contribuyendo a mantener la población en el territorio, sobre todo rurales, en mayor grado que las empresas mercantiles, consideradas éstas como el sector empresarial convencional.

Estos análisis se realizan tanto para las emigraciones totales como para las emigraciones de mujeres y jóvenes, por las dificultades sociolaborales de estos colectivos en la actualidad y por su interés estratégico en el afianzamiento de la población al territorio, manteniendo el relevo intergeneracional en las actividades económicas locales.

La comparación de las sociedades cooperativas con las empresas mercantiles se lleva a cabo para fundamentar el establecimiento de estrategias y políticas de impulso del cooperativismo de manera específica, en el caso de que el comportamiento de estas organizaciones de economía social difiera del de las empresas mercantiles.

La metodología empleada para alcanzar el objetivo se desarrolla mediante análisis exploratorios de la proporción de cooperativas, de empresas mercantiles y del porcentaje de emigraciones. Además, se han aplicado técnicas de econometría espacial, en concreto el Índice Global de Moran, para la detección de la autocorrelación o dependencia espacial de las citadas variables, y regresiones espaciales. Todo ello se realiza a nivel municipal para el caso de Andalucía.

La estructura seguida en el presente trabajo, tras esta introducción donde se ha expuesto el interés actual del tema, consiste en un segundo apartado en el que se describe la metodología aplicada, seguido de un tercero donde se exponen los resultados y discusión derivados de su aplicación. Finalmente, se presentan las consideraciones finales del presente estudio, recogiendo las principales conclusiones y contribuciones de la investigación. Entre los resultados obtenidos destaca que el cooperativismo podría estar contribuyendo a fijar la población al territorio y, en mayor grado, en los de carácter rural, con especial incidencia en mujeres y jóvenes. 


\section{Metodología}

Para dar respuesta al objetivo planteado, tras un análisis descriptivo de las variables, se aplican técnicas de econometría espacial que tienen en cuenta la ubicación de los datos en un determinado espacio geográfico de las variables objeto de estudio: gráficos de análisis de distribución y autocorrelación espaciales y aplicación de regresiones espaciales.

La elección de esta metodología se justifica por la existencia de literatura acerca de que el cooperativismo guarda relación con el entorno en el que trabaja (García-Gutiérrez, 1999; Buendía y García, 2003; Coque, 2005; Mozas y Bernal, 2006; Cuñat y Coll, 2007; Puentes y Velasco, 2009; Calvo y González, 2011; Demoustier, 2011; Draperi, 2014; Guzmán, Santos y Barroso, 2016; Pérez y Valiente, 2017). Por otro lado, debido a que Andalucía cuenta con una diversidad territorial significativa, se plantea la hipótesis de que el cooperativismo pueda no seguir una distribución aleatoria en esta región, sino que responda a un patrón de comportamiento que guarde relación con el territorio. Este comportamiento debe ser tenido en cuenta a la hora de analizar estas organizaciones de economía social, desde la perspectiva territorial, y aplicar políticas y estrategias para su impulso y desarrollo.

Siguiendo la premisa anterior, se determina si existe o no autocorrelación espacial de las variables objeto de estudio (de la proporción de cooperativas, de la proporción de las empresas mercantiles y del porcentaje de emigraciones); se trata de analizar si lo que sucede en un determinado punto del espacio, en este caso en un municipio, y lo que ocurre en otros (municipios) está relacionado (Anselin, 1988), pudiendo deberse a las características socioeconómicas y territoriales de los entornos. En concreto, se mide el nivel de dependencia de un municipio respecto a los demás que están cercanos. De otra forma, la autocorrelación espacial determina el grado en que determinados factores, características o actividades de un territorio, son similares a las de territorios próximos (Goodchild, 1987).

A su vez, esta autocorrelación espacial puede ser positiva o negativa. La autocorrelación espacial positiva tiene lugar cuando valores altos (o bajos) de una variable aleatoria se distribuyen de manera agrupada en el espacio, es decir, presentan un comportamiento similar; por el contrario, la autocorrelación espacial negativa se da cuando unidades territoriales próximas presentan valores muy diferentes entre sí (Cliff y Ord, 1981).

Para contrastar la existencia de autocorrelación espacial se emplea el Índice Global de Moran (1948), que establece como hipótesis nula la ausencia de autocorrelación espacial o la distribución de la variable de forma aleatoria entre las diferentes áreas objeto de estudio. Este índice se expresa a continuación: 


$$
I=\frac{n}{S_{0}} \frac{\sum_{i=j}^{n} \sum_{j=1}^{n} w_{i j}\left(y_{i}-\bar{y}\right)\left(y_{j}-\bar{y}\right)}{\sum_{i=1}^{n}\left(y_{i}-\bar{y}\right)^{2}} \text { para } i \neq j,
$$

siendo $y_{i}$ la variable analizada en el territorio $i, \bar{y}$ la media de la variable $y, w_{i j}$ elemento o coeficiente de ponderación de la matriz $\mathrm{W}$ denominada de conexión, de contigüidad o de pesos geográfi$\cos$, que determina qué municipios son vecinos entre sí, $S_{0}$ es el sumatorio de los pesos espaciales, es decir, $S_{0}=\sum_{i=j}^{n} \sum_{j=1}^{n} w_{i j}$ para $i \neq j$, y $n$ es el tamaño muestral.

La matriz W está formada por valores de unos y ceros, según exista contigüidad o no entre determinadas zonas geográficas, tal que $\mathrm{w}_{\mathrm{ij}}=1$ si las regiones $i$ y $j$ tienen una frontera común, $y \mathrm{w}_{\mathrm{ij}}=0$ si no (Moreno y Vayá, 2000).

En este caso, en la aplicación de la / de Moran, se ha considerado el tipo Rook (con retardo espacial de primer orden), que considera que $i$ y $j$ son municipios vecinos si comparten algún borde o lado (y no vértices). Este índice (I) es normalizado (Duncan, 1991): $Z(I)=\frac{I-E(I)}{\sqrt{V(I)}} \sim N(0,1)$, siendo $E(I)$ el valor medio de I y $V(I)$ su desviación típica. Cuando el valor de $Z(I)$ es estadísticamente significativo se confirma la existencia de autocorrelación espacial.

La / de Moran puede variar entre 1 y -1, donde 0 indica la inexistencia de autocorrelación espacial (distribución aleatoria de la variable), es decir de un patrón de comportamiento definido, mientras que valores cercanos a - 1 o 1 significa la existencia de autocorrelación espacial negativa o positiva respectivamente.

Esta existencia de autocorrelación o dependencia espacial invalida las estimaciones por la metodología tradicional de Mínimos Cuadraros Ordinarios (MCO u OLS por sus siglas en inglés). Siguiendo a Anselin (1992), hay que destacar que pueden existir dos tipos de dependencia o autocorrelación espacial: la sustantiva, que tiene lugar cuando el valor que toma la variable está influenciado por el valor de éstas en las unidades vecinas (lo que se determina, en el presente trabajo, mediante la aplicación a las variables objeto de estudio del Índice o la / de Moran descrito arriba); y la del término error, que incumple el supuesto de la no correlación de los errores. Es necesario, por tanto, en el caso de que se produzca, considerar la dependencia espacial en el análisis de regresión, para obtener una estimación eficiente del modelo.

Suponiendo que exista autocorrelación espacial de la variable dependiente, es decir, teniendo en cuenta que se produce dependencia o autocorrelación espacial sustantiva, el modelo es el siguiente:

$$
y=r W y+X \beta+\varepsilon,
$$

siendo r el parámetro de rezago espacial (término Spatial Lag), Wla matriz de pesos geográficos, $X$ la matriz de covarianzas y $\varepsilon$ el vector de errores no correlacionados. El valor de y en un punto concreto del espacio se relaciona con el resto de valores con el término autorregresivo espacial $r W y$ 
(modelo Spatial Lag); mientras que si dicho efecto ocurre en los términos de error (modelo Spatial Error), el modelo se representa como sigue:

$$
\begin{gathered}
y=X \beta+\varepsilon, \\
\varepsilon=\lambda W \varepsilon+\xi,
\end{gathered}
$$

siendo $\lambda$ el grado de autocorrelación de los errores dada una matriz de pesos geográficos $\mathrm{W}$ (Sánchez Peña, 2012).

La estimación de los modelos se ha realizado con el software Geoda que proporciona, una vez realizada la estimación por $\mathrm{MCO}$, diagnósticos espaciales para determinar qué tipo de autocorrelación se está produciendo y decidir el modelo adecuado que recoja dicho efecto.

En lo que se refiere a las variables dependientes, como variables proxy de la fijación de la población al territorio, se consideran las siguientes:

- Porcentaje de emigraciones: número de personas que han emigrado sobre el total de la población.

- Porcentaje de emigraciones de personas jóvenes ${ }^{1}$ : número de personas entre 16 y 39 años de edad que emigran sobre el total de población con la misma edad.

- Porcentaje de emigraciones de mujeres: número de mujeres que emigran sobre el total de mujeres.

Se han obtenido estas variables para los diferentes municipios andaluces (un total de 770), a partir de las Estadísticas de Variaciones Residenciales y datos del Padrón Municipal de Habitantes del Instituto de Estadística y Cartografía de Andalucía (IECA), para el año 2015.

El hecho de considerar las emigraciones, y no el saldo migratorio, es debido a que en el presente trabajo se pone la atención en la capacidad para fijar la población al territorio de los residentes en el mismo, sin que ello signifique que no se trabaje con dicha variable, el saldo migratorio, en futuras líneas de investigación. En la última década en España, ante la crisis económica iniciada en 2008 y la incapacidad de los mercados laborales para absorber la fuerza de trabajo existente, una cifra significativa de personas jóvenes y, en parte, cualificadas, ha emigrado en busca de un proyecto de vida que comienza con la inclusión en el mercado laboral. Ello implica una pérdida de potencial de determinados territorios para crear dinámicas económicas.

Por otro lado, también se ha contado con las variables número de cooperativas sobre el total de empresas y número de empresas mercantiles (sociedades anónimas y sociedades limitadas) sobre el total de empresas, expresadas en porcentaje (denominadas como proporción de cooperativas y pro-

1.- Dada la no disponibilidad de los datos de emigraciones de personas entre 16 y 35 años de edad, en este estudio se consideran jóvenes a las personas con edades comprendidas entre 16 y 39 años. 
porción de empresas mercantiles), también para el año 2015 y a nivel municipal. Estos datos proceden del Directorio de Empresas y Establecimientos del IECA.

Además, al objeto de determinar si existen diferencias en las emigraciones entre municipios rurales y no rurales, se ha añadido una variable ficticia denominada municipio o territorio rural: como se ha señalado anteriormente, Según la Ley de Desarrollo Sostenible del Medio Rural (LDSMR), se considera municipio rural de pequeño tamaño a aquéllos que tienen una población inferior a 5.000 habitantes; esta variable tendrá valor 1 si se trata de un municipio rural de pequeño tamaño (en adelante municipio rural) y 0 en caso de que sea un municipio no rural (Gráfico 1).

Para los modelos de regresión, tanto las variables dependientes (porcentaje de emigraciones totales, porcentaje de emigraciones de jóvenes y porcentaje de emigraciones de mujeres) como las explicativas (proporción de cooperativas y proporción de empresas mercantiles) se expresan en logaritmos.

Hay que destacar que, con la metodología descrita, en el presente trabajo no se pretende identificar un modelo que explique el comportamiento de las emigraciones en Andalucía, sino determinar si el cooperativismo podría estar influyendo en éstas y si existe algún componente espacial en la distribución de los valores de las variables objeto de estudio, es decir, si la vecindad entre municipios afecta a la distribución del cooperativismo (y de las empresas mercantiles) así como a la de emigraciones.

\section{Resultados}

\subsection{Análisis exploratorios}

Partiendo de que, según el criterio de la LDSMR, más del $66 \%$ del territorio de Andalucía puede ser considerado como rural (516 municipios), se tiene lo siguiente: se confirma el significativo carácter de ruralidad que presenta Andalucía, ya que la media del porcentaje de emigraciones totales es superior en el ámbito rural (4,45\% frente al $3,57 \%$ de los municipios no rurales), ocurriendo lo mismo con las emigraciones de jóvenes (6,93\% y 5,25\% para los municipios rurales y no rurales, respectivamente) y de mujeres (el ámbito rural representa el 4,58\% frente al valor de 3,47\% del no rural) (Tabla 1).

Por otro lado, aunque para el porcentaje de emigraciones totales no suceda, para el de jóvenes y mujeres los valores máximos son superiores en los municipios rurales, existiendo para estas últimas, las emigraciones de mujeres, una diferencia significativa con el ámbito no rural. Esto pone en evidencia la problemática actual en el medio rural andaluz a la que se enfrentan mujeres y jóvenes, colectivos que ven agravadas sus dificultades de acceso al mercado laboral, en estos territorios, con respecto a los municipios no rurales (Tabla 1). 


\section{Tabla 1. Estadísticos principales de las variables de emigraciones}

\begin{tabular}{|l|ccc|ccc|ccc|}
\hline & \multicolumn{3}{|c|}{$\%$ de emigraciones } & \multicolumn{3}{c|}{$\%$ emigraciones de jóvenes } & \multicolumn{3}{c|}{$\%$ emigraciones de mujeres } \\
& Rural & No rural & Total & Rural & No rural & Total & Rural & No rural & Total \\
\hline Media & 4.45 & 3.57 & 4.16 & 6.93 & 5.25 & 6.37 & 4.58 & 3.47 & 4.21 \\
Mediana & 4.08 & 3.08 & 3.76 & 6.27 & 4.80 & 5.72 & 4.07 & 2.99 & 3.68 \\
Mínimo & 0.64 & 1.25 & 0.64 & 0 & 1.96 & 0 & 0 & 1.11 & 0 \\
Máximo & 14.73 & 16.72 & 16.72 & 20 & 19.77 & 20 & 25 & 16.17 & 25 \\
Desv. típica & 2.14 & 1.75 & 2.06 & 3.21 & 2.05 & 2.99 & 2.53 & 1.68 & 2.34 \\
Coef. variación & 0.48 & 0.49 & 0.49 & 0.46 & 0.39 & 0.47 & 0.55 & 0.48 & 0.55 \\
Asimetría & 1.51 & 2.38 & 1.71 & 1.34 & 1.86 & 1.56 & 2.39 & 2.39 & 2.50 \\
Exc. de Curtosis & 3.31 & 12.09 & 4.79 & 2.30 & 8.65 & 3.44 & 10.99 & 12.20 & 12.17 \\
\hline
\end{tabular}

FUENTE: Elaboración propia a partir de datos del IECA.

En cuanto a la proporción de empresas cooperativas y la de mercantiles, aunque el peso del cooperativismo en los municipios andaluces, en términos generales, es bajo ya que de media tan sólo el $1,88 \%$ de las empresas son cooperativas-, hay que destacar lo siguiente: en el ámbito rural la media para el caso de las cooperativas (2,23\%) es superior a la del no rural $(1,17 \%)$, no ocurriendo lo mismo con las empresas mercantiles (la media en los municipios rurales es del 22,01\% mientras que en los no rurales su valor asciende hasta el 29,77\%) (Tabla 2). Esto manifiesta un comportamiento diferenciado del cooperativismo, organizaciones que destacan en los municipios rurales frente a las empresas convencionales.

El comportamiento diferente de las cooperativas frente a las organizaciones convencionales puede apreciarse también mediante el coeficiente de variación: en términos totales, éste manifiesta mayor variabilidad o dispersión respecto al valor medio para las cooperativas, ya que ha sido mucho mayor que el de las empresas mercantiles (1,44 frente al 0,42 respectivamente). Por otro lado, el coeficiente de asimetría para la proporción de cooperativas es positivo $(4,56)$, lo que indica un sesgo hacia la derecha en su distribución; es decir, la mayoría de municipios cuenta con una proporción muy baja o nula de cooperativas.

Para la proporción de empresas mercantiles, tanto la asimetría como el coeficiente de Curtosis, están muy próximos a 0 , lo que significa que, al existir la misma cantidad de valores a los dos lados de la media, existen indicios de que su distribución a lo largo del territorio andaluz sigue un comportamiento normal (Tabla 2). Ello implica cierto patrón de comportamiento de mayor uniformidad en Andalucía para el caso del sector empresarial convencional que para el del cooperativismo y, por tanto, una mayor heterogeneidad en la distribución de este último frente al mayor carácter homogéneo que presentan las empresas convencionales en su ubicación territorial. 


\section{Tabla 2. Estadísticos principales de las variables sobre la proporción de empresas (cooperativas y mercantiles)}

\begin{tabular}{|l|cccccc|}
\hline & \multicolumn{3}{|c}{ Proporción sociedades cooperativas (en \%) } & \multicolumn{3}{c|}{ Proporción empresas mercantiles (en \%) } \\
& Rural & No rural & Total & Rural & No rural & Total \\
\hline Media & 2.23 & 1.17 & 1.88 & 22.01 & 29.77 & 24.59 \\
Mediana & 1.43 & 0.92 & 1.14 & 21.25 & 28.93 & 25 \\
Mínimo & 0 & 0 & 0 & 0 & 9.69 & 0 \\
Máximo & 35.29 & 5.44 & 35.29 & 65 & 61.77 & 65 \\
Desv. típica & 3.20 & 0.98 & 2.72 & 10.62 & 7.95 & 10.47 \\
Coef. variación & 1.43 & 0.83 & 1.44 & 0.48 & 0.27 & 0.42 \\
Asimetría & 3.88 & 1.74 & 4.56 & 0.28 & 0.75 & 0.11 \\
Exc. de Curtosis & 27.03 & 3.70 & 37.65 & 0.44 & 1.65 & 0.51 \\
\hline
\end{tabular}

FUENTE: Elaboración propia a partir de datos del IECA.

A partir de todo lo anterior, cabe destacar que son los municipios rurales los que mayor proporción de emigraciones presentan, lo que se podría traducir en mayores dificultades para fijar la población al territorio; no obstante, son también estos municipios los que cuentan con mayor proporción de cooperativas. Esta idea conduce, por tanto, a plantear la siguiente pregunta: ¿sería mayor el porcentaje de emigraciones si la proporción de cooperativas fuese menor? De otra forma, ¿podría estar contribuyendo el cooperativismo a fijar la población en el territorio andaluz? Para intentar dar respuesta a esta cuestión, se han aplicado análisis de regresiones espaciales.

\subsection{Regresiones espaciales}

Previamente al análisis de MCO y de regresiones espaciales (Spatial Lag y Spatial Error), se ha trabajado con el Índice de Moran, aplicado a las variables objeto de estudio, para determinar si existe o no dependencia espacial de los valores que toman las variables en un determinado municipio respecto a los contiguos o cercanos.

Los resultados obtenidos del Índice o la / de Moran, respecto a la proporción de cooperativas y a la proporción de empresas mercantiles, ponen de manifiesto la existencia de autocorrelación espacial para ambos casos; es decir, a nivel general, el número de empresas -en términos relativos- de un determinado municipio se ve influenciado por el número de éstas que existen en municipios contiguos. Esto se concluye al examinar los pseudo $\mathrm{p}$-valores asociados a los valores estandarizados de la / de Moran $(Z(I))$, los cuales son inferiores a 0,05 , lo que lleva a rechazar la hipótesis nula de aleatoriedad espacial en las variables objeto de análisis. Por ello, los resultados son estadísticamente significativos pudiéndose confirmar la existencia de autocorrelación espacial, tanto en la proporción de cooperativas como en el caso de empresas mercantiles o convencionales (Gráficos 2 y 3 ). 


\section{Gráfico 2. Diagrama de dispersión de la / de Moran global de la proporción de cooperativas y significatividad}

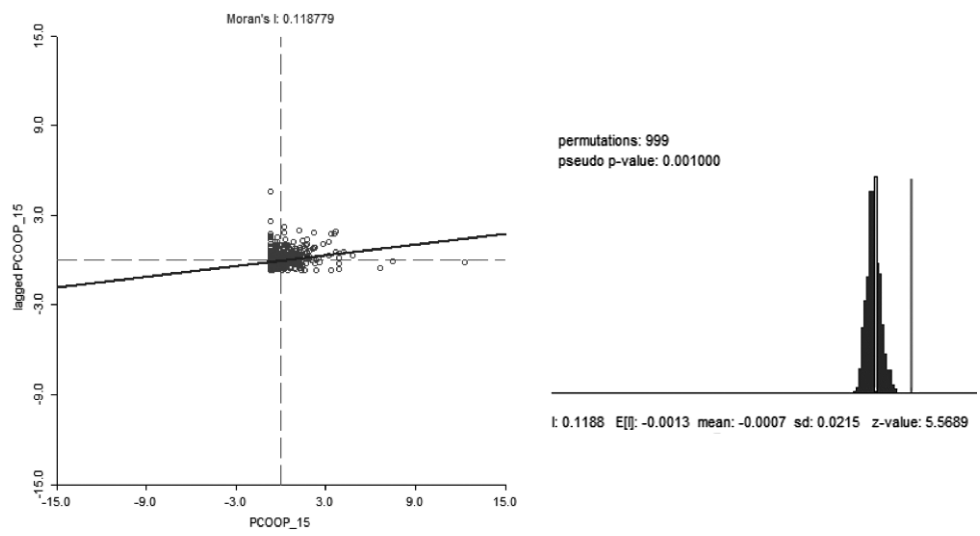

FUENTE: Elaboración propia a partir de datos del IECA.

Además, esta autocorrelación espacial es positiva, lo que indica que valores altos (o bajos) de la proporción de cooperativas en un determinado municipio implica valores altos (o bajos) de esta misma variable en municipios vecinos o contiguos. Lo mismo ocurre para la proporción de sociedades mercantiles. Esto pone de manifiesto el posible efecto de sinergias que podría estar generándose por la interacción de empresas y agentes territoriales de los diferentes municipios andaluces que son contiguos, lo que supone una oportunidad para producir efectos contagio o de arrastre en la aplicación de políticas y estrategias territoriales, en este caso, enfocadas a fijar la población al territorio.

Si se compara la / de Moran de la proporción de cooperativas con el de la proporción de empresas mercantiles, hay que destacar que el valor obtenido es mayor en las segundas $(0,28$ frente a 0,11 para el caso de las cooperativas), lo que podría indicar la existencia de mayores sinergias positivas que en el caso del cooperativismo; es decir, que valores altos de la proporción de empresas mercantiles, en un determinado municipio, inciden en que se produzca una alta proporción de estas empresas en los municipios contiguos. Esto se podría traducir en que la cultura empresarial cooperativa se difunde hacia territorios vecinos en menor grado que la del sector empresarial convencional.

En lo que respecta a las variables referidas a las emigraciones, ocurre lo mismo: existe autocorrelación o dependencia espacial de los valores -ya que los pseudo $p$-valores asociados a los valores estandarizados de la $I$ de Moran $(Z(I))$ son inferiores a 0,05 -. Esto significa que el valor que tome tanto el porcentaje total de emigraciones en general, como el de mujeres y jóvenes en particular, se ve afectado por los valores de los municipios contiguos (Gráfico 4). 


\section{Gráfico 3. Diagrama de dispersión de la / de Moran global de la proporción de empresas mercantiles y significatividad}

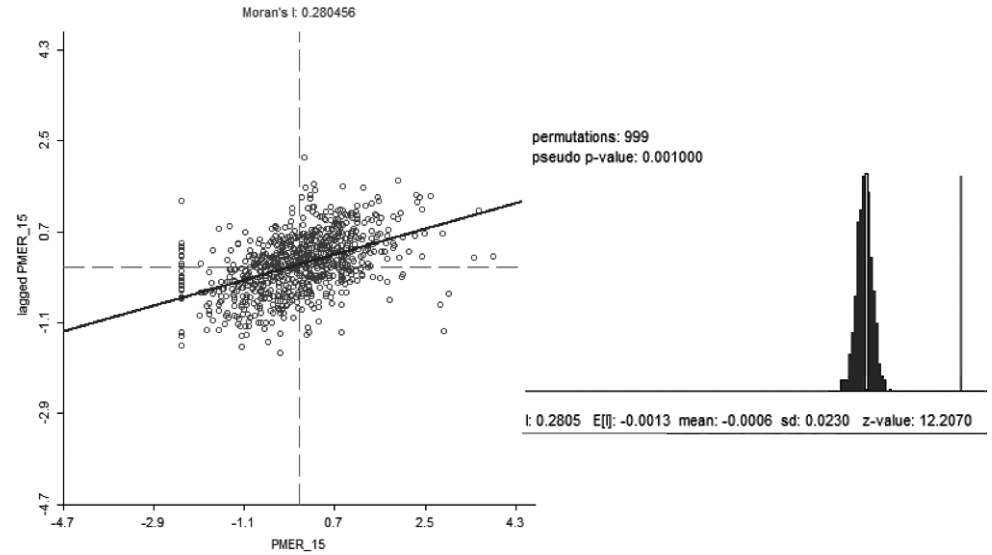

FUENTE: Elaboración propia a partir de datos del IECA.

Atendiendo a lo anterior, a la hora de aplicar estrategias y políticas encaminadas a fijar la población en el territorio, en este caso mediante el impulso y desarrollo del cooperativismo, este efecto de sinergia positiva (autocorrelación espacial positiva) supone una ventaja en el sentido de que, poniendo el foco de atención en un determinado municipio, dichos efectos pueden transmitirse a municipios contiguos. Sin embargo, para poder afirmar dicha idea, es necesario determinar si, efectivamente, existen indicios acerca de que el cooperativismo podría estar contribuyendo a fijar la población al territorio andaluz. Es por ello que se aplican análisis de regresiones considerando estos efectos de autocorrelación o dependencia espacial a la hora de estimar los modelos.

De la aplicación de la metodología de MCO y su interpretación, se obtiene que el modelo es estadísticamente significativo ( $p$-valor asociado al estadístico $\mathrm{F}$ es inferior a 0,05 ). En lo que respecta a la significatividad individual de las variables, el logaritmo de la proporción de cooperativas sí es significativo; por el contrario, no ocurre lo mismo con el logaritmo de la proporción de empresas mercantiles. Además, el coeficiente obtenido para la variable de las cooperativas es negativo, es decir, a medida que la proporción de cooperativas se incrementa, el porcentaje de emigraciones disminuye, lo que se traduce en que estas organizaciones de economía social podrían estar contribuyendo a fijar la población al territorio, medido esto con el porcentaje de emigraciones. En cuanto a la variable ficticia sobre territorio rural, también ha resultado significativa (Tabla 3), lo que indica la existencia de diferencias entre territorios rurales y no rurales. 


\section{Gráfico 4. Diagrama de dispersión de la / de Moran global del porcentaje de emigraciones y significatividad}

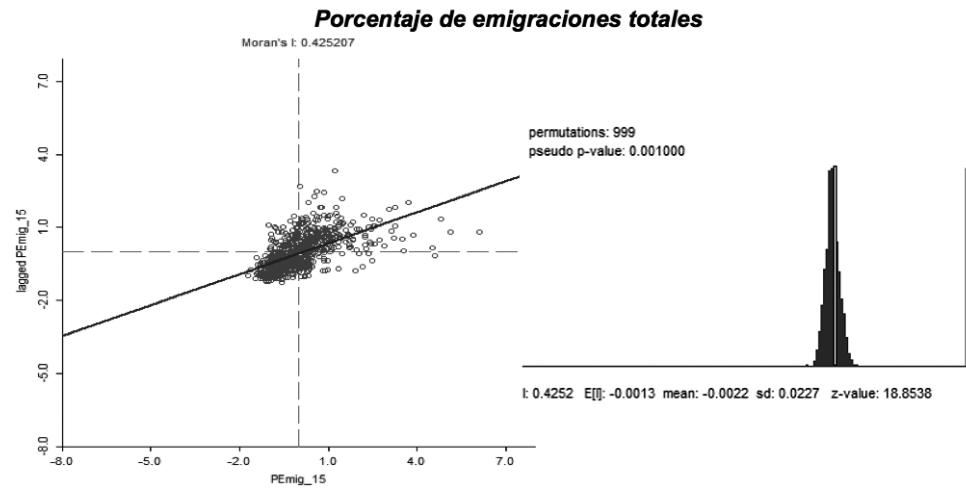

Porcentaje de emigraciones de jóvenes
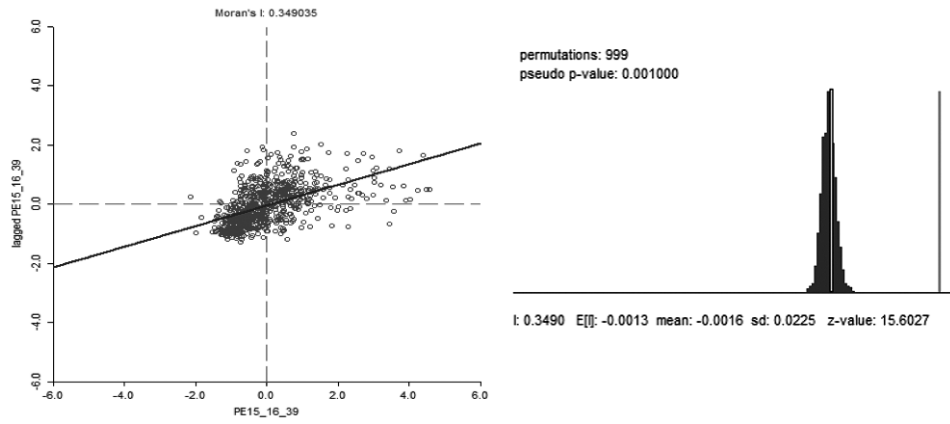

I: 0.3490 E[n: -0.0013 mean: -0.0016 sd: 0.0225 z-value: 15.6027

Porcentaje de emigraciones de mujeres

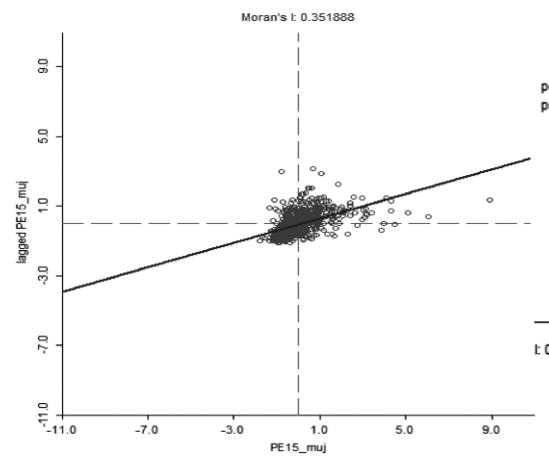

permutations: 999

pseudo p-value: 0.001000

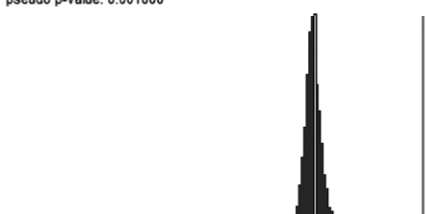

t. 0.3519 E[n: -0.0013 mean: -0.0022 sd: 0.0221 z-value: 16.0536

FUENTE: Elaboración propia a partir de datos del IECA. 
Sin embargo, los test de dependencia espacial indican que el modelo estimado por MCO no es el más adecuado: de los diferentes test, el que presenta mayor valor del multiplicador de Lagrange, siendo significativo, es el que mide la existencia de dependencia o autocorrelación espacial sustantiva (Lagrange Multiplier lag). Esto demuestra y refuerza la presencia de autocorrelación espacial, ya detectada previamente mediante la aplicación del Índice de Moran a las respectivas variables. Por tanto, debe estimarse un modelo Spatial Lag para tener en cuenta este efecto (Tabla 3).

Lo idoneidad del modelo Spatial Lag frente al de MCO se ve reflejado tanto en el Criterio de Información de Akaike como en el de Schwarz, ya que ambos valores disminuyen al pasar del modelo MCO al Spatial Lag. Además, el valor del Likelihood Ratio Test, que compara la nulidad del modelo de MCO con el modelo alternativo, en este caso Spatial Lag, ha sido significativo al $99 \%$, lo que indica que, efectivamente, el modelo que recoge el efecto de la autocorrelación o dependencia espacial es más adecuado frente al modelo de regresión tradicional MCO (Tabla 3).

\section{Tabla 3. Resultados análisis de regresión MCO (OLS) y regresión Spatial Lag para el porcentaje de emigraciones}

\begin{tabular}{|c|c|c|c|}
\hline Var. depend: In (\% emigraciones 2015) & OLS (MCO) & Spatial lag & Spatial error \\
\hline Constante & $1.25505^{\star \star \star}$ & $0.403739^{\star \star \star}$ & $1.27889^{\star \star *}$ \\
\hline $\operatorname{Ln}(\%$ cooperativas $)$ & $-0.118219^{\star \star *}$ & $-0.0611194^{\star \star *}$ & $-0.0456771^{\star * *}$ \\
\hline Ln (\% empresas mercantiles) & -0.025933 & -0.0106171 & -0.00544765 \\
\hline Municipio rural (1=sí, 0=no) & $0.282034^{\star * *}$ & $0.179317^{* \star *}$ & $0.156657^{\star \star *}$ \\
\hline Término espacial lag & & $0.657209^{\star * *}$ & \\
\hline Término espacial lambda & & & $0.675971^{* * *}$ \\
\hline R-squared / Pseudo R-squared & 0.086739 & 0.4675 & 0.4475 \\
\hline Adjusted R-squared/Spatial pseudo R-squared & 0.083162 & 0.1687 & 0.0828 \\
\hline F-statistic & $24.2509^{\star \star *}$ & & \\
\hline Log likelihood & -454.507 & -292.804 & -300.676 \\
\hline Akaike info criterion & 917.013 & 595.608 & 609.354 \\
\hline Schwarz criterion & 935.599 & 618.839 & 627.939 \\
\hline \multicolumn{4}{|l|}{ Diagnostics for spatial dependence: } \\
\hline Moran's I (error) & $20.5749^{\star \star \star}$ & & \\
\hline Lagrange Multiplier (lag) & $471.0692^{* * *}$ & & \\
\hline Robust LM (lag) & $60.7535^{\star \star \star}$ & & \\
\hline Lagrange Multiplier (error) & $413.9102^{\star * *}$ & & \\
\hline Robust LM (error) & $3.5945^{\star}$ & & \\
\hline Lagrange Multiplier (SARMA) & $474.6637^{\star \star *}$ & & \\
\hline Likelihood Ratio Test & & $323.4056^{\star \star *}$ & $307.6595^{\star \star *}$ \\
\hline
\end{tabular}

${ }^{* * *}$ significativo al nivel de confianza del $99 \%,{ }^{* *}$ al $95 \%$ y ${ }^{*}$ al $90 \%$

FUENTE: Elaboración propia a partir de Geoda. 
Atendiendo al modelo final propuesto, Spatial Lag, considerando como variable dependiente el logaritmo del porcentaje de emigraciones, el logaritmo de la proporción de cooperativas como variable explicativa es significativa a un nivel de confianza del $99 \%$; de lo contrario, la variable de la proporción de empresas mercantiles no es significativa para explicar la variabilidad del porcentaje de emigraciones. Además, la variación porcentual de la proporción de cooperativas tiene un efecto inverso en la del porcentaje de emigraciones, lo que indica que existen indicios acerca de que el cooperativismo podría estar contribuyendo a fijar la población al territorio.

Por otro lado, hay que destacar que el carácter de ruralidad del municipio influye en las decisiones sobre emigraciones de dichos municipios andaluces (la variable es estadísticamente significativa a un nivel de confianza del 99\%): existe un diferencial positivo en el porcentaje de emigraciones en los municipios considerados como rurales con respecto a municipios no rurales (Tabla 3). Esto refuerza el resultado ya obtenido en los análisis exploratorios sobre el mayor grado de emigraciones que tienen lugar en los territorios rurales andaluces.

En lo que respecta a las variables dependientes de las emigraciones de jóvenes y de la de mujeres, ocurre lo mismo que para el caso anterior: los test de diagnóstico de dependencia espacial indican que el mejor modelo es el que recoge el efecto de la autocorrelación espacial de carácter sustantivo de las observaciones, es decir, el modelo Spatial Lag. Cuando se estima dicho modelo, tanto el Criterio de Información de Akaike como el de Schwarz disminuyen, por lo que la estimación mejora frente al modelo MCO (Tablas 4 y 5 ).

Por otro lado, en este caso, también ha sido significativo el logaritmo de la proporción de cooperativas como variable explicativa, tanto de la variabilidad del porcentaje de emigraciones de jóvenes como el de mujeres (a un nivel de confianza del 99\%) y, además, existe una relación inversa entre la variable dependiente y la explicativa. Esto indica que un incremento porcentual de la proporción de cooperativas puede incidir en una disminución porcentual de la tasa de emigraciones. Sin embargo, esto no ocurre con el logaritmo de la proporción de empresas mercantiles que no ha resultado ser significativa.

A lo anterior hay que añadir que, en ambos casos, tanto para el cooperativismo como para el sector empresarial convencional, la variable que identifica a los municipios rurales también ha sido significativa a un nivel de confianza del $99 \%$, lo que indica que las emigraciones de estos colectivos, mujeres y jóvenes, se ven afectadas, incrementándose por el carácter de ruralidad de los municipios. 


\section{Tabla 4. Resultados análisis de regresión MCO (OLS) y regresión Spatial Lag para el \% de emigraciones de jóvenes}

\begin{tabular}{|c|c|c|c|}
\hline Var. depend: In (\% emigraciones 2015 jóvenes) & OLS (MCO) & Spatial lag & Spatial error \\
\hline Constante & $1.70153^{\star \star \star}$ & $0.71464^{\star \star \star}$ & $1.69124^{* \star *}$ \\
\hline Ln (\% cooperativas) & $-0.0882424^{* * *}$ & $-0.0488396^{\star \star *}$ & $-0.030609^{*}$ \\
\hline Ln (\% empresas mercantiles) & -0.0345642 & -0.015812 & -0.0095214 \\
\hline Municipio rural (1=sí, 0=no) & $0.288929^{\star * \star}$ & $0.198155^{\star \star *}$ & $0.176011^{\star * *}$ \\
\hline Término espacial lag & & $0.562181^{\star * *}$ & \\
\hline Término espacial lambda & & & $0.577837^{\star \star \star}$ \\
\hline R-squared / Pseudo R-squared & 0.092181 & 0.3538 & 0.332079 \\
\hline Adjusted R-squared/Spatial pseudo R-squared & 0.088626 & 0.1512 & 0.0871 \\
\hline F-statistic & $25.9269^{\star \star \star}$ & & \\
\hline Log likelihood & -424.793 & -325.663 & 181.8264 \\
\hline Akaike info criterion & 857.586 & 661.326 & 675.76 \\
\hline Schwarz criterion & 876.172 & 684.558 & 694.346 \\
\hline \multicolumn{4}{|l|}{ Diagnostics for spatial dependence: } \\
\hline Moran's I (error) & $15.5012^{\star \star \star}$ & & \\
\hline Lagrange Multiplier (lag) & $282.2572^{\star \star \star}$ & & \\
\hline Robust LM (lag) & $55.9529^{\star \star \star}$ & & \\
\hline Lagrange Multiplier (error) & $413.9102^{\star \star \star}$ & & \\
\hline Robust LM (error) & $7.8904^{\star \star \star}$ & & \\
\hline Lagrange Multiplier (SARMA) & $290.1475^{\star * *}$ & & \\
\hline Likelihood Ratio Test & & $198.2604^{\star \star *}$ & $181.8264^{* * *}$ \\
\hline
\end{tabular}

${ }^{* * *}$ significativo al nivel de confianza del $99 \%,{ }^{* *}$ al $95 \%$ y ${ }^{*}$ al $90 \%$

FUENTE: Elaboración propia a partir de Geoda.

Por tanto, hay que destacar que, atendiendo a estos resultados, se confirma que en los municipios rurales se agravan las dificultades para fijar la población al territorio, sobre todo respecto a los colectivos de mujeres y jóvenes. Por otro lado, el cooperativismo incide de manera inversa en las emigraciones de los municipios rurales, lo que le convierte en una oportunidad para aminorar los problemas de despoblamiento de estos territorios mediante, entre otras acciones, la creación de empleo sostenible y de calidad. 
Tabla 5. Resultados análisis de regresión MCO (OLS) y regresiones espaciales para el porcentaje de emigraciones de mujeres

\begin{tabular}{|c|c|c|c|}
\hline Var. depend: In (\% emigraciones 2015 de mujeres) & OLS (MCO) & Spatial lag & Spatial error \\
\hline Constante & $1.22073^{\star \star \star}$ & $0.428654^{* * *}$ & $1.23145^{\star \star \star}$ \\
\hline $\operatorname{Ln}(\%$ cooperativas $)$ & $-0.110878^{* * *}$ & $-0.0620488^{* * *}$ & $-0.0486705^{* *}$ \\
\hline Ln (\% empresas mercantiles) & -0.0227945 & -0.00695369 & 0.00093937 \\
\hline Municipio rural (1=sí, 0=no) & $0.31114^{* * *}$ & $0.21013^{\star \star *}$ & $0.191707^{\star \star *}$ \\
\hline Término espacial lag & & $0.614535^{\star \star *}$ & \\
\hline Término espacial lambda & & & $0.632828^{* * *}$ \\
\hline R-squared / Pseudo R-squared & 0.085836 & 0.4075 & 0.332079 \\
\hline Adjusted R-squared/Spatial pseudo R-squared & 0.082256 & 0.1507 & 0.0827 \\
\hline F-statistic & $23.9748^{* * *}$ & & \\
\hline Log likelihood & -505.694 & -378.207 & -385.0931 \\
\hline Akaike info criterion & 1019.39 & 766.415 & 778.186 \\
\hline Schwarz criterion & 037.97 & 789.647 & 796.772 \\
\hline \multicolumn{4}{|l|}{ Diagnostics for spatial dependence: } \\
\hline Moran's I (error) & $18.0428^{\star \star *}$ & & \\
\hline Lagrange Multiplier (lag) & $364.5564^{* * *}$ & & \\
\hline Robust LM (lag) & $50.4013^{\star \star \star}$ & & \\
\hline Lagrange Multiplier (error) & $317.8658^{\star \star *}$ & & \\
\hline Robust LM (error) & $3.710^{*}$ & & \\
\hline Lagrange Multiplier (SARMA) & $368.2671^{* * *}$ & & \\
\hline Likelihood Ratio Test & & $254.9735^{\star \star *}$ & $241.202264^{* * *}$ \\
\hline
\end{tabular}

${ }^{* * *}$ Significativo al nivel de confianza del 99\%, ${ }^{* *}$ al $95 \%$ y *al $90 \%$

FUENTE: Elaboración propia a partir de Geoda. 


\section{Consideraciones finales}

En el presente trabajo se ha determinado si las cooperativas en Andalucía, organizaciones de economía social representativas en la región, podrían estar contribuyendo a fijar la población en el territorio, en mayor grado que el sector empresarial convencional (empresas mercantiles, representadas éstas por las sociedades anónimas y sociedades limitadas).

Partiendo de la idea de que las cooperativas guardan relación con el entorno en el que trabajan y que el territorio andaluz presenta una diversidad territorial significativa, la metodología empleada para dar respuesta a dicho objetivo ha sido de especial interés. Esta metodología ha consistido en la aplicación del Índice Global de Moran, para determinar la existencia de autocorrelación espacial, y el desarrollo de modelos de regresión espacial; una metodología que tiene en cuenta la ubicación de los valores que toma las variables en el espacio geográfico, en este caso, Andalucía. Es decir, se ha partido de la hipótesis de que el cooperativismo no sigue una distribución aleatoria en la región andaluza, sino que responde a un patrón de comportamiento que guarda relación con el territorio (lo que se ha confirmado mediante la aplicación del Índice de Moran).

Se ha identificado que las emigraciones (variable tomada como proxy de la capacidad para fijar la población al territorio) especialmente de mujeres y jóvenes, son mayores en los municipios rurales, en concreto los de pequeño tamaño, que en los no rurales. Además, son en estos municipios rurales de pequeño tamaño, donde existe una mayor proporción de cooperativas (en comparación con los no rurales). Esto se ha podido comprobar mediante los diferentes análisis exploratorios realizados.

Atendiendo a este resultado acerca de que en el medio rural las emigraciones son mayores y también la proporción de cooperativas, se ha planteado la cuestión de si un menor cooperativismo podría implicar un mayor grado de emigraciones; de otra forma, si el cooperativismo podría estar contribuyendo a fijar la población al territorio.

Los análisis de regresión han demostrado que la variable "proporción de cooperativas", como explicativa de la variabilidad del porcentaje de emigraciones en el territorio andaluz, ha sido significativa y, además, presentan ambas una relación inversa, lo que podría ser un indicio acerca de que el cooperativismo sí podría estar contribuyendo a fijar la población al territorio.

Por su parte, la variable que representa la proporción de empresas mercantiles no ha sido significativa, no influyendo, por tanto, en la variabilidad de las emigraciones en el territorio andaluz. Lo mismo se ha obtenido cuando dichas variables dependientes utilizadas han sido el porcentaje de emigraciones de mujeres y el de jóvenes: la proporción de cooperativas incide de manera inversa en dichas tasas de emigraciones, no sucediendo lo mismo con la proporción de empresas mercantiles. 
Por tanto, dado que, por un lado, en los municipios rurales andaluces se agravan las dificultades para fijar la población al territorio, sobre todo de mujeres y jóvenes y, por otro, el cooperativismo incide de manera inversa en las emigraciones de los municipios rurales, la creación de empleo sostenible y de calidad mediante estas sociedades de economía social, las cooperativas, pueden suponer una oportunidad para aminorar los problemas de despoblamiento de estas zonas.

Otro de los resultados obtenidos es que la cultura empresarial cooperativa podría estar difundiéndose hacia territorios vecinos en menor grado que la del sector empresarial convencional (como ha demostrado el Índice de Moran). Si el cooperativismo podría estar contribuyendo a fijar la población al territorio (según los resultados de las regresiones espaciales), aplicar políticas y estrategias específicas que logren disminuir las emigraciones en los territorios rurales -impulsando el desarrollo de las sociedades cooperativas para la creación de empleo sostenible y de calidad y, de esta forma, difundir en mayor grado la cultura cooperativa-, se convierte al mismo tiempo en un reto y oportunidad para el territorio andaluz.

Desde otra perspectiva, se podrían fomentar las sinergias empresariales entre el cooperativismo y las empresas convencionales, aprovechando la oportunidad que éstas últimas ofrecen de difundir su cultura hacia territorios vecinos en mayor grado que el cooperativismo. De esta forma, se podría lograr al mismo tiempo, mediante la posible intercooperación empresarial, difundir los principios y valores del cooperativismo en el territorio andaluz y contribuir a fijar la población al territorio (impulsando la creación de empleo sostenible y de calidad, sobre todo en las zonas rurales).

La necesidad de estrategias y políticas específicas para impulsar el cooperativismo en Andalucía se ha podido determinar, además de lo anterior, mediante los análisis exploratorios o descriptivos: el cooperativismo presenta un comportamiento más heterogéneo en el territorio andaluz que el sector empresarial convencional (como han mostrado los coeficientes de variación en los análisis descriptivos), lo que justifica esta necesidad de otorgarle un tratamiento más específico en la región.

Por otro lado, hay que destacar que el presente trabajo no está exento de limitaciones. En primer lugar, se ha realizado un análisis de carácter estático, para un determinado momento del tiempo (año 2015), que supone una limitación para analizar la dinámica evolutiva que han seguido tanto las sociedades cooperativas como las emigraciones en Andalucía. En segundo lugar, se ha tomado como variable proxy o indicativa de la dificultad para fijar la población al territorio el porcentaje de emigraciones; sin embargo, hay que tener en cuenta que se pueden dar otros factores: puede ocurrir que el porcentaje de emigraciones sea bajo o nulo como consecuencia de un envejecimiento de la población considerable, por lo que, en este caso, el despoblamiento o la dificultad para fijar la población al territorio vendría motivada, más bien, por la falta de crecimiento demográfico. Además, debe tenerse en cuenta que, como se ha señalado al comienzo del presente estudio, el objetivo no ha sido identificar un modelo que explique el comportamiento de las emigraciones en Andalucía, sino determinar la existencia o no de cierto componente espacial y relacional entre esta variable y el cooperativismo. 
A partir de las limitaciones señaladas surgen, a su vez, futuras líneas de investigación, destacando las siguientes: analizar la dinámica evolutiva entre el cooperativismo y las emigraciones, al objeto de identificar la relación que han seguido en el tiempo; y considerar otros factores indicativos de la capacidad para fijar la población al territorio como la ya citada tasa de crecimiento demográfico.

Además, hay que añadir que sería de especial interés identificar clústeres espaciales, en Andalucía, sobre el comportamiento de la relación entre estas dos variables, determinando, al mismo tiempo, qué tipo de cooperativismo es el que predomina, especialmente desde la perspectiva del empleo. Esto permitiría considerarlos, a cada grupo o conglomerado obtenido, como focos para la actuación, aplicando políticas y estrategias para el impulso del cooperativismo y así evitar el despoblamiento de las zonas rurales(ya que se ha demostrado que lo que sucede en un municipio, en lo que concierne a estas variables, está afectado por lo que ocurre en municipios contiguos). Ello permitiría generar un efecto arrastre o contagio a territorios vecinos a partir de esta actuación en los focos que se identifiquen.

\section{Referencias bibliográficas}

ANSELIN, L. (1988): Spatial Econometrics: Methods and Models, Dordrech: Kluwer.

ANSELIN, L. (1992): Spatial Data Analysis with GIS: An Introduction to Application in the Social Science, Santa Barbara: University of California Santa Barbara, Technical Report, 92-10.

BUENDIA, I. \&COTE, A. (2014): "Desarrollo territorial rural y cooperativas: un análisis desde las políticas públicas", Cuadernos de Desarrollo Rural, 11(74): 35-54. DOI: https://doi.org/10.11144/Javeriana.CRD11-74.dtrc

BUENDIA, I. \& GARCIA J.V. (2003): Cooperativismo y desarrollo local, Madrid: Editorial Centro de Estudios Ramón Areces.

BURGUÉS, A., MARTIN, S. \& SANTA CRUZ, I. (2013): "La relación entre cooperativas transformadoras y desigualdades sociales en los territorios", Scripta Nova, Revista Electrónica de Geografía y Ciencias Sociales, 427 (4). DOI: http://dx.doi.org/10.1344/sn2013.17.14911.

CABANES, M. \& GÓMEZ, J.D. (2014): "Economía social y Soberanía Alimentaria. Aportaciones de las cooperativas y asociaciones agroecológicas de producción y consumo al bienestar de los territorios", CIRIEC-España, Revista de Economía Pública, Social y Cooperativa, 82, 127-154. Disponible en: http://www.ciriec-revistaeconomia.es/banco/CIRIEC_8205_Cabanes_Gomez.pdf. 
CALVO, R. \& GONZÁLEZ, J. (2011): "La creación de empresas de economía social en el modelo de desarrollo local: reflexiones sobre la divergencia actual del caso valenciano", REVESCO, Revista de Estudios Cooperativos, 104, 7-37. DOI: https://doi.org/10.5209/rev_REVE.2011.v104.1.

CARAVACA, I., GONZALEZ, G. \& MENDOZA, A. (2007): "Indicadores de dinamismo, innovación y desarrollo: su aplicación en ciudades pequeñas y medias de Andalucía", Boletín de la Asociación de Geógrafos Españoles, 43, 131-154. Disponible en: https://www.agegeografia.es/ojs/index.php/bage/article/view/585/539.

CLIFF, A.D. \&ORD, J.K. (1981): Spatial processes: models \& applications, Vol. 44, London: Pion.

COLLETIS, G. \&PECQUEUR, B. (1993): "Intégration des espaces et quasi intégration des firmes: vers de nouvelles rencontres productives?", Revue d'Economie Régionale et Urbaine, 3: 485-508.

COQUE, J. (2005): Compartir soluciones: las cooperativas como factor de desarrollo en zonas desfavorecidas, Madrid: Consejo Económico y Social.

CUÑAT, R. \& COLL, V. (2007): “¿Contribuyen las cooperativas de reciente creación al desarrollo local? Una visión desde los principios cooperativistas", Revista Venezolana de Economía Social, 13: 8-29.

DEMOUSTIER, D. (2011): "Les concepts philosophiques et sociaux de l'ESS dans ses rapports à l'activité économique et aux territoires". En: A. Ndiaye (dir.), Économie sociale et solidaire: animation et dynamiques des territoires, Paris: L'Harmattan, 47-60.

DRAPERI, J.F. (2014): Comprendre l'économie sociale. Fondements et enjeux, Paris: Dunod.

ESTEBAN, M.L., PÉREZ, F.J. \& GARGALLO, A. (2018): "Áreas rurales y cooperativas: iniciativas de mujeres para el desarrollo", REVESCO, Revista de Estudios Cooperativos, 127: 116-138. DOI: 10.5209/REVE.58397.

GARCÍA, J.L. (Dir.) (2004): Las Cuentas de la Economía Social. El Tercer Sector en España, Madrid: Civitas/ Fundación ONCE.

GARCÍA-GUTIÉRREZ, C. (1999): "Cooperativismo y desarrollo local", REVESCO, Revista de Estudios Cooperativos, 68: 33-46.

GOODCHILD, M. (1987): "A spatial analytical perspective on geographical information systems", International Journal of Geographical Information System, 1: 327-334.

GUZMÁN, C., SANTOS, F.J. \& BARROSO, M. (2016): "Cooperativismo, factor empresarial y desarrollo económico: propuesta de un modelo teórico de enlace", REVESCO, Revista de Estudios Cooperativos, 122: 110-134.

JULIÁ, J.F. \& MARÍ, S. (2002): "Agricultura y desarrollo rural. Contribuciones de las cooperativas agrarias", CIRIEC-España, Revista de Economía Pública, Social y Cooperativa, 41: 25-52. 
MÉNDEZ, R., MELERO, A. \& CALATRAVA, A. (2008): "Desarrollo territorial policéntrico y ciudades intermedias: recursos productivos y dinámicas económicas locales en Andalucía", Estudios Geográficos, 69(265): 637-663.

MORENO, R. \& VAYÁ, E. (2000): Técnicas econométricas para el tratamiento de datos espaciales: La Econometría Espacial, Edicions de la Universitat de Barcelona, UB 44 Manuals.

MOZAS, A. \& BERNAL, E. (2006): "Desarrollo territorial y economía social", CIRIEC-España, Revista de Economía Pública, Social y Cooperativa, 55: 125-140.

NOVKOVIC, S. (2008): "Defining the co-operative difference", The Journal of Socio-Economics, 37 (6): 2168-2177. Doi:10.1016/j.socec.2008.02.009.

PÉREZ, M.C. \& VALIENTE, L. (2017): "La localización sectorial del cooperativismo: una aproximación a nivel territorial español", REVESCO, Revista de Estudios Cooperativos, 123: 198-224. DOI: https://doi.org/10.5209/REVE.54916.

PÉREZ, M.C. \& VALIENTE, L. (2019): "Aproximación al perfil y calidad del empleo generado por las sociedades cooperativas andaluzas", REVESCO, Revista de Estudios Cooperativos, 130: 122148. DOI: 10.5209/REVE.60989.

PITA, M.F. \& PEDREGAL, B. (Coords.) (2011): Tercer Informe de Desarrollo Territorial de Andalucía. Sevilla, Secretariado de Publicaciones de la Universidad de Sevilla. Disponible en http://grupo.us.es/giest/.

PUENTES, R. \& VELASCO, M. (2009): "Importancia de las sociedades cooperativas como medio para contribuir al desarrollo económico, social y medioambiental, de forma sostenible y responsable", REVESCO, Revista de Estudios Cooperativos, 99: 104-129.

SÁNCHEZ, P., GALLARDO, R. \& CEÑA, F. (2014): "El medio rural andaluz frente a la crisis económica: un análisis de los factores de resiliencia territorial", Economía Agraria y Recursos Naturales, 14(1):27-56. DOI: 10.7201/earn.2014.01.02.

SÁNCHEZ-PEÑA, L. (2012): "Alcances y límites de los métodos de análisis espacial para el estudio de la pobreza urbana", Papeles de Población, 18 (72): 147-179.

SILVA, R. (2002): "Estrategias de inserción de las áreas rurales en la economía mundial. Una aproximación desde Andalucía", Boletín de la A.G.E., 33: 103-131.

SOLER, M. (2009): El contexto socioeconómico de la agricultura ecológica: la evolución de los sistemas agroalimentarios, Universidad de Sevilla. Disponible en http://institucional.us.es/compromiso/libreconf/docs/sistemas.pdf.

TORRES, F.J. \& OJEDA, J.F. (2004): "Pobreza en Andalucía", SEMATA, Ciencias Sociais e Humanidades, 16: 79-99. 
TORTOSA, J.M., DE PABLO, J. \& URIBE, J. (2018): "Innovación social como motor del desarrollo local y el emprendimiento social", Equidad y Desarrollo, 31: 11-25. DOI: http://dx.doi.org/10.19052/ed.4416.

VILALLONGA, A. (2002): "Los nuevos yacimientos de empleo: una oportunidad para crear empleo y satisfacer nuevas necesidades sociales", Scripta Nova, Revista Electrónica de Geografía y Ciencias, v. 6, no 119.

ZOIDO, F., PEDREGAL, B., PITA, M.F., TORRES, F.J. \& FERNÁNDEZ-TABALES, A. (2009): "Medición del desarrollo territorial en las escalas de detalle. Aplicación a Andalucía". En: J. Farinós, J. Romero y J. Salom (eds.), Cohesión e inteligencia territorial. Dinámicas y procesos para una mejor planificación y toma de decisiones, Valencia: Publicaciones de la Universitat de Valencia, 245-265. 